Virginia Commonwealth University VCU Scholars Compass

2009

\title{
Magnetism in assembled and supported silicon endohedral cages: First-principles electronic structure calculations
}

\author{
R. Robles \\ Virginia Commonwealth University \\ Shiv N. Khanna \\ Virginia Commonwealth University, snkhanna@vcu.edu
}

Follow this and additional works at: http://scholarscompass.vcu.edu/phys_pubs

Part of the Physics Commons

Robles, R., Khanna, S.N. Magnetism in assembled and supported silicon endohedral cages: First-principles electronic structure calculations. Physical Review B, 80, 115414 (2009). Copyright (C 2009 American Physical Society.

\section{Downloaded from}

http://scholarscompass.vcu.edu/phys_pubs/71

This Article is brought to you for free and open access by the Dept. of Physics at VCU Scholars Compass. It has been accepted for inclusion in Physics Publications by an authorized administrator of VCU Scholars Compass. For more information, please contact libcompass@vcu.edu. 


\title{
Magnetism in assembled and supported silicon endohedral cages: First-principles electronic structure calculations
}

\author{
R. Robles and S. N. Khanna* \\ Department of Physics, Virginia Commonwealth University, Richmond, Virginia 23284-2000, USA \\ (Received 4 June 2009; revised manuscript received 14 August 2009; published 15 September 2009)
}

\begin{abstract}
First principles electronic structure calculations on a free $\mathrm{CrSi}_{12}$ cluster, a $\left(\mathrm{CrSi}_{12}\right)_{2}$ dimer, and $\mathrm{CrSi}_{12}$ clusters supported on $\mathrm{Si}(111)$ surfaces have been carried out within a gradient corrected density functional formalism using a supercell approach. The ground state of $\mathrm{CrSi}_{12}$ is a $\mathrm{Cr}$ centered hexagonal biprism of $\mathrm{Si}$ atoms in which the $\mathrm{Cr}$ spin moment is completely quenched. As two $\mathrm{CrSi}_{12}$ motifs are brought together, they form different composite units depending on initial direction of approach and, in most cases, the composite cluster is found to have a net spin moment. Cluster assemblies obtained by depositing $\mathrm{CrSi}_{12}$ motifs on a $\mathrm{Si}(111)$ surface exhibit similar finite spin moments for several initial directions of approach. An analysis of the electronic states shows that the origin of the magnetic moment lies in those $\mathrm{Cr} d$-states that do not mix with silicon $s p$ states. The studies suggest the possibility of forming silicon-based magnetic semiconductors through such assemblies.
\end{abstract}

DOI: 10.1103/PhysRevB.80.115414

PACS number(s): 73.22.-f, 75.50.Pp, 75.75.+a

\section{INTRODUCTION}

One of the grand challenges in cluster science is to synthesize nanomaterials where size selected clusters serve as the building blocks. ${ }^{1,2}$ As the electronic, magnetic and chemical properties of clusters change with size and composition, it offers the potential of designing materials with a precise control over properties. Indeed, several groups are actively pursuing this direction by trying to synthesize large amounts of cluster motifs in beams and depositing size selected clusters to make the cluster materials. ${ }^{1-3}$ Since the intensity of different clusters in beams depends on their stability, the ideal cluster motifs are those that are stable as they have larger intensity in beams as well as the greater potential to maintain their identity. The discovery of fullerenes and the development of fullerides ${ }^{4-6}$ demonstrated the viability of synthesizing such materials. In addition to bare $\mathrm{C}_{60}$ cage, templated endohedral metallofullerenes like $\mathrm{Sc}_{3} \mathrm{~N} @ \mathrm{C}_{80}$ have attracted attention as the endohedral motif stabilizes the cage through charge transfer, and doped cages like $\mathrm{Gd}_{3} \mathrm{~N} @ \mathrm{C}_{80}$ are being sought for applications as MRI contrast agents. ${ }^{7}$ Since carbon and silicon belong to the same group of the periodic chart, these developments have raised the question if the findings in fullerenes could be translated to silicon, the material most widely used in semiconductor industry. In particular, if by impregnating the silicon cage with a metal atom, one could stabilize the cage and yet maintain the magnetic moment of the metal atom. This would allow the possibility of injecting a spin into the semiconductor, a first step toward silicon based spintronics devices. The exploration of this possibility started a flurry of activity on the stability and properties of $\mathrm{MSi}_{n}$ clusters containing transition metal atoms. An important development in this direction came from experiment by Hiura, Kanayama, and co-workers, ${ }^{8}$ who demonstrated that clusters such as $\mathrm{WSi}_{12}$ are very stable. Assuming that each silicon atom contributes one electron and the tungsten atom contributes 6 valence electrons, the authors suggested that the enhanced stability of $\mathrm{WSi}_{12}$ could be a consequence of the 18 electron rule, well known in chemis- try. $\mathrm{Cr}$ and $\mathrm{W}$ belong to the same group, but $\mathrm{Cr}$ has the highest spin moment in the $3 d$ series. Theoretical studies in our group ${ }^{9-11}$ and by other groups ${ }^{12-15}$ on $\mathrm{CrSi}_{12}$ and analogous clusters indeed found them to be exceptionally stable. The $\mathrm{Cr}$ atom in the cluster is encapsulated in a silicon cage with a hexagonal biprism structure with D6h symmetry. The atomic spin moment on $\mathrm{Cr}$ was, however, completely quenched inside the cage. The theoretical findings were confirmed by the experimental negative ion photodetachment spectra on $\mathrm{CrSi}_{n}^{-}$clusters. ${ }^{16}$ Further studies ${ }^{10,11}$ on $\mathrm{MSi}_{12}$ cages containing other $3 d$ transition metal atoms yielded similar reduction of the transition metal moments. Thus, while the interaction between the metal atom and the cage stabilized the cluster, the resulting magnetic moments were completely quenched. It will be ideal if the spin moment on the $\mathrm{Cr}$ sites could be somehow recovered as the large intensity of $\mathrm{CrSi}_{12}$ in beams allows larger intensity for cluster deposition.

In this paper, we report a rather surprising finding that indicates that it may indeed be possible to recover spin moments in silicon cages for their use in spintronics. Our findings are based on theoretical investigations where we explored the nature of the cluster assembled material that would result if the $\mathrm{CrSi}_{12}$ cages were randomly landed on a bulk silicon surface to make a material. We found that, while the individual motifs are nonmagnetic, depending on the landing site and orientation, the clusters acquire net spin moments. The magnetic moments also emerge as two building units are brought together. While the surface deposition opens the possibility of making silicon based magnetic semiconductors, the composite clusters obtained by bringing together two $\mathrm{CrSi}_{12}$ motifs may lead to interesting transport properties in molecular electronics. ${ }^{17}$ The findings are particularly significant as assemblies through deposition of endohedral silicon cages avoid two problems. First, the solubility of transition metals in $\mathrm{Si}$ is extremely low and it is difficult to implant magnetic impurities in bulk Si. Assembling the stable cages ensures a high concentration of transition metal. Second, the ion implantation in bulk is often lim- 
ited by formation of magnetic clusters. As the magnetic atom is located in the silicon cage, the formation of metallic clusters is avoided. The recent evidence of room temperature ferromagnetism in ion-implanted bulk silicon suggests that the cluster assembled material may exhibit interesting behaviors. $^{18}$

In Sec. II, we describe our theoretical approach, while Sec. III presents results and a discussion of findings. Section IV contains a summary of the main findings.

\section{THEORETICAL METHOD}

The theoretical studies were carried out using the density functional theory package Vienna $a b$ initio simulation package (VASP), ${ }^{19}$ and the projector augmented wave method. ${ }^{20,21}$ The exchange correlation contributions were included using the generalized gradient approximation (GGA) proposed by Perdew et al. ${ }^{22}$ Wave functions were expanded using a plane wave basis set with a cutoff energy of $300 \mathrm{eV}$. The Brillouin zone was sampled at the $\Gamma$ point. The magnetic moments at the local sites were calculated by projecting the wave functions on spherical waves within atomic spheres and integrating the resulting local spin-polarized densities of states up to the Fermi level.

\section{RESULTS AND DISCUSSION}

We first investigated the ground state of an isolated neutral and anionic $\mathrm{CrSi}_{12}$ cluster for which previous theoretical investigations ${ }^{9}$ and negative ion photodetachment data ${ }^{16}$ are available. The present studies did find $\mathrm{CrSi}_{12}$ to have a hexagonal bi-prism structure with $\mathrm{Cr}$ sandwiched between two $\mathrm{Si}_{6}$ hexagons. The calculated $\mathrm{Cr}-\mathrm{Si}$ and $\mathrm{Si}-\mathrm{Si}$ bond lengths of $2.62 \AA$ and $2.37 \AA$ were close to the previously calculated values of $2.70 \AA$ and $2.38 \AA$, respectively. The ground state was a singlet, confirming the previously found quenching of the spin-magnetic moment. We also determined the ground state of $\mathrm{CrSi}_{12}^{-}$and calculated the vertical transition energy in going from the anion to the neutral species. The calculated value of $3.04 \mathrm{eV}$ is in reasonable agreement with the experimental value of $3.18 \mathrm{eV}$ from photodetachment spectroscopy. ${ }^{16}$

We next consider how the properties of a $\mathrm{CrSi}_{12}$ motif change as two units are brought together to form a composite cluster. The investigations included four possible scenarios, as shown in Fig. 1.

Figure 1(a) refers to the case where the two clusters approach each other with hexagonal faces aligned. The second situation [Fig. 1(b)] is similar to the previous one, but with one cluster rotated by 30 degrees in the alignment direction. In the third and four situations [Figs. 1(c) and 1(d)], a quadrangular face of one cluster is aligned with a hexagonal one in the other cluster. In each case, the atoms were relaxed in the direction of forces till the forces dropped below a threshold value of $0.01 \mathrm{eV} / \AA$. Figure 1 shows the equilibrium configurations and the relative energy of the various configurations obtained by comparing their total energies. Also shown are the total spin magnetic moment $\left(M_{T}\right)$ and the local spin moment at the metal sites. In three cases, the composite

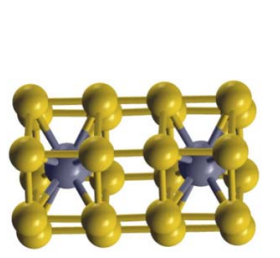

(a)

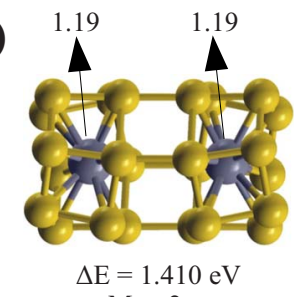

$\mathrm{M}_{\mathrm{T}}=2 \mu_{\mathrm{B}}$

(b)
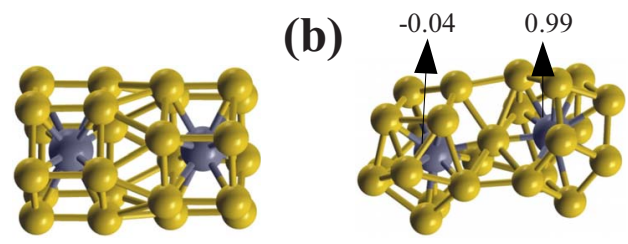

$\Delta \mathrm{E}=0.000 \mathrm{eV}$

$\mathrm{M}_{\mathrm{T}}=2 \mu_{\mathrm{B}}$

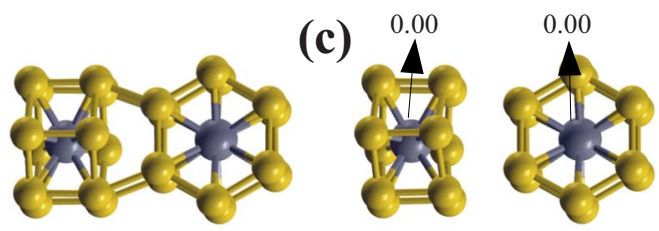

$\Delta \mathrm{E}=1.534 \mathrm{eV}$ $\mathrm{M}_{\mathrm{T}}=0 \mu_{\mathrm{B}}$

(d)
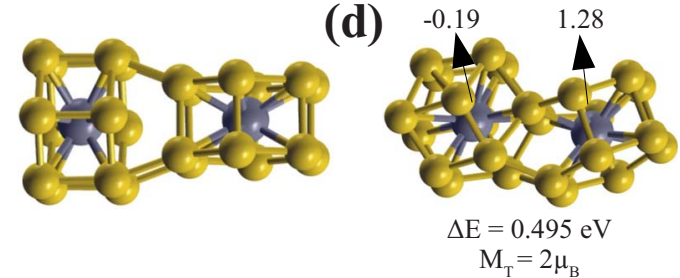

FIG. 1. (Color online) Starting (left column) and equilibrium (right column) configurations of two $\mathrm{CrSi}_{12}$ clusters brought together. For each of the four configurations we show the relative energy $(\Delta E)$, total spin moment $\left(M_{T}\right)$, and local spin moment at the $\mathrm{Cr}$ atoms $\left(\right.$ in $\mu_{B}$ ). Dark gray (yellow) spheres represent chromium (silicon) atoms.

cluster had a net moment of $2 \mu_{B}$ and nonzero local spin moments at the $\mathrm{Cr}$ sites. A closer analysis of the resulting geometry shows that the appearance of the magnetic moment occurs in those cases where the $\mathrm{Si}$ atoms of one cage bond to $\mathrm{Si}$ atoms of the second cage. The formation of these new $\mathrm{Si}-\mathrm{Si}$ bonds reduces the coupling between the $\mathrm{Si}$ and the metal atoms and distorts the structure of the clusters. This is what happens in the first situation [Fig. 1(a)], where both $\mathrm{Cr}$ atoms have a local spin moment of $1.19 \mu_{B}$. It is also the case for the right $\mathrm{Cr}$ atom in the second situation [Fig. 1(b)]. However, in the left $\mathrm{Cr}$ atom of this configuration new $\mathrm{Si}-\mathrm{Cr}$ bonds are formed with $\mathrm{Si}$ atoms from the cage on the right, and therefore the local magnetic moment at this site is close to zero $\left(-0.04 \mu_{B}\right)$. Similar analysis can be made for the remaining two cases. Note that the quenching of the $\mathrm{Cr}$ moment in isolated cages is due to the formation of bonds with $\mathrm{Cr}$ that enhances electron kinetic energy over the exchange energy favoring parallel spins. The weakening of the $\mathrm{Cr}-\mathrm{Si}$ bonds as new $\mathrm{Si}-\mathrm{Si}$ bonds are formed then favors the exchange enhancement through aligned spins. We will return to this point later. 


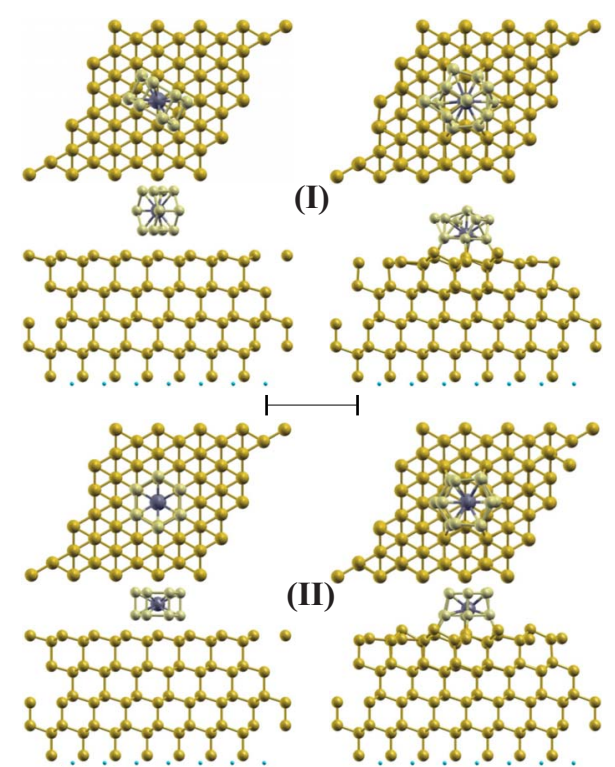

FIG. 2. (Color online) Starting (left column) and equilibrium (right column) configurations of a $\mathrm{CrSi}_{12}$ cluster on $\mathrm{Si}(111)$. Two different situations (I and II) are shown and, for each one, a top and a side view are presented. Dark gray spheres represent chromium atoms, while silicon is represented by dark yellow spheres in the surface and lighter ones in the supported cluster. Small blue spheres represent hydrogen atoms, used for passivation.

One way to enhance $\mathrm{Si}-\mathrm{Si}$ bonding is to deposit the clusters on surfaces and, hence, we examined the effect of landing the nonmagnetic cluster on to a silicon surface. In this work we focus on a $\mathrm{Si}(111)$ surface. The infinite surface was modeled by using a supercell with nine layers in the [111] direction, each layer containing 16 atoms. An extra layer of hydrogen was added on the bottom to passivate this surface. The five top layers were allowed to relax, while the rest were fixed to the bulk values. The relaxation was carried on till the forces were less than $0.01 \mathrm{eV} / \AA$. To examine the effect of deposition, $\mathrm{CrSi}_{12}$ clusters were deposited starting from various initial orientations. Here, we present results on four such possibilities of clusters on the top side of the slab, both with a hexagonal or a quadrangular face on top of the surface and in several positions. As before, the clusters approached the surface and the relaxations were carried out by calculating the forces on the cluster and moving the atoms in the direction of forces. It is important to point out that our objective here is not to find the most stable configuration, but to simulate the experimental situation where the material is formed by deposition of clusters from the beam on to the $\mathrm{Si}(111)$ surface. Figures 2 and 3 present several of these investigations showing the starting and the final configurations along with the supercell used to mimic the infinite surface. While the principal quantity of interest is the local spin moment at the $\mathrm{Cr}$ site, we were also interested in the formation energy $E_{F}$ defined as

$$
E_{F}=E\left[\mathrm{CrSi}_{12}\right]+E[\mathrm{Si}(111)]-E_{T},
$$

where $E\left[\mathrm{CrSi}_{12}\right]$, and $E[\mathrm{Si}(111)]$ are the total energies of the bare $\mathrm{CrSi}_{12}$ cluster and $\mathrm{Si}(111)$ surface, while $E_{T}$ is the total

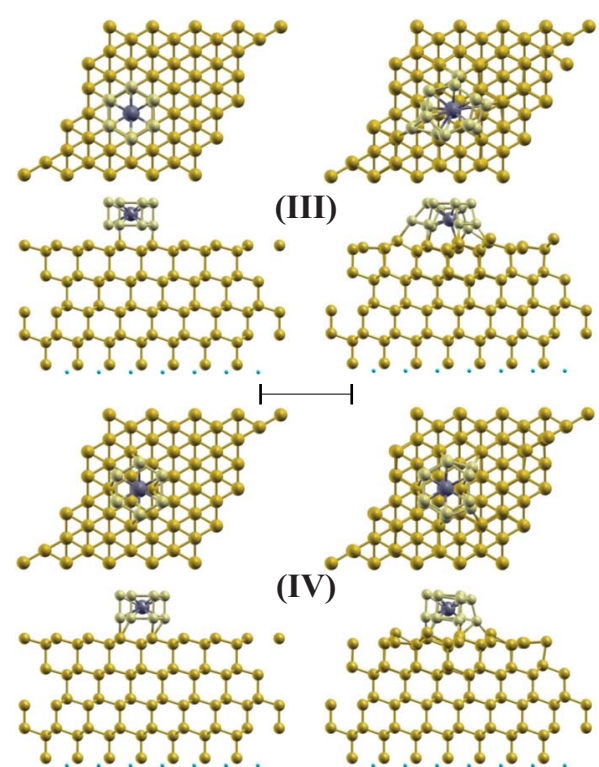

FIG. 3. (Color online) Same as in Fig. 2 but for configurations III and IV.

energy of the combined system. The information on $E_{F}$ and the spin moments for the four configurations are summarized in Table I. The lowest energy configuration (I) corresponds to the $\mathrm{Si}_{12}$ cage of the $\mathrm{CrSi}_{12}$ cluster approaching the surface with a quadrangular face parallel to the surface, as indicated in Fig. 2. The relaxation results in the total distortion of the silicon cage and in the coordination of the $\mathrm{Cr}$ atom to one of the $\mathrm{Si}$ atoms of the surface. The $\mathrm{Cr}$ moment is then completely quenched. In configuration II the cage is far less distorted, but a bond between the $\mathrm{Cr}$ atom with a $\mathrm{Si}$ atom in the surface is also formed and the magnetic moment is quenched as well. The situation is different in the two cases of Fig. 3 . In configuration III the cage is heavily distorted and a bond is formed between the $\mathrm{Cr}$ atom and the surface but, unlike in configuration I, some $\mathrm{Si}-\mathrm{Cr}$ bonds corresponding to the cage are broken. Consequently, the $\mathrm{Cr}$ magnetic moment is not quenched, presenting a value of $1.43 \mu_{B}$. Finally, in configuration IV the cage is less distorted but, since the $\mathrm{Cr}$ atom do not form new bonds as in case II, the magnetic moment reaches a value of $1.76 \mu_{B}$. It is worth to note that, regardless the distortion of the cluster upon deposition, in all cases the $\mathrm{Cr}$ atom remains encapsulated.

To further ascertain that the results obtained above are not affected by the size of the supercell, supplementary calculations were carried out where we increased the supercell to

TABLE I. Formation energy $\left(E_{F}\right)$ and local spin moment at the $\mathrm{Cr}$ site for four different configurations.

\begin{tabular}{lcc}
\hline \hline & $\begin{array}{c}E_{F} \\
(\mathrm{eV})\end{array}$ & $\mu(\mathrm{Cr})\left(\mu_{B}\right)$ \\
\hline I & 9.81 & 0.00 \\
II & 9.50 & 0.00 \\
III & 8.18 & 1.43 \\
IV & 7.21 & 1.76 \\
\hline \hline
\end{tabular}




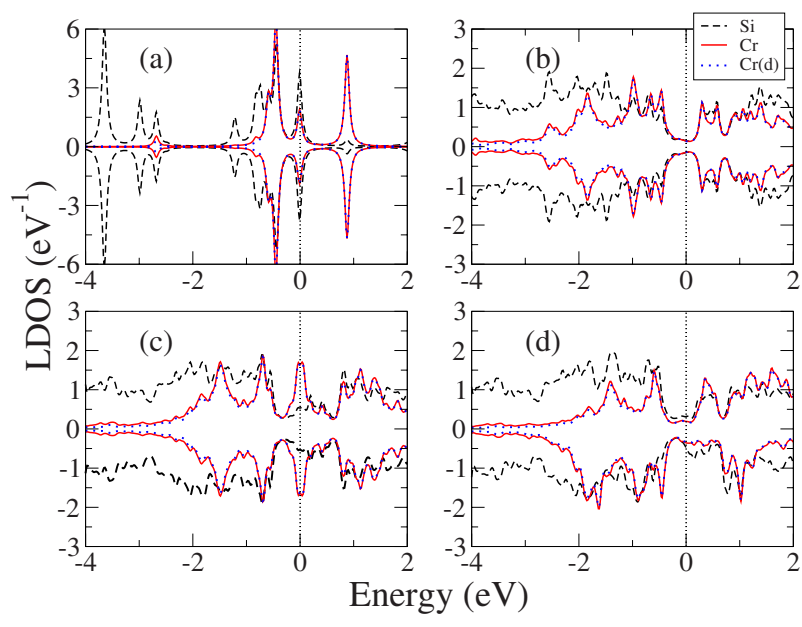

FIG. 4. (Color online) Densities of states of $\mathrm{Cr}$ and $\mathrm{Si}$ atoms of the isolated and supported $\mathrm{CrSi}_{12}$ clusters. (a) Isolated cluster. (b) Supported cluster in configuration (i). (c) and (d) Supported cluster in configuration (III) in the paramagnetic (c) and magnetic (d) situations. Dashed lines show Si states, while solid and dotted lines show $\mathrm{Cr}$ total and $d$ states, respectively.

include 25 silicon atoms on each layer. Also the sampling of the Brillouin zone was tested by including six $k$ points in the calculations. The two investigations were carried out for the geometry shown in III. In both cases, the resulting geometry was very close to the one shown in Fig. 3, and the spin moment at the $\mathrm{Cr}$ site changed by less than $2 \%$, affirming that the results presented here are not limited by numerical approximations.

A detailed analysis of the electronic states provides insight into the microscopic mechanism underlying the appearance of magnetic moment. Figure 4(a) shows the density of states (DOS) in an isolated $\mathrm{CrSi}_{12}$ cluster. Note that a Gaussian with a half width of $0.05 \mathrm{eV}$ has been placed at each electronic state to obtain an uniform DOS. The DOS was projected on $\mathrm{Si}$ and $\mathrm{Cr}$ sites to probe the densities of states at $\mathrm{Si}$ and $\mathrm{Cr}$ sites, and the $d$-density of states at the $\mathrm{Cr}$ atom. Two features draw particular attention. First, the $d$ states of $\mathrm{Cr}$ exhibit a strong maxima around $0.5 \mathrm{eV}$ below the Fermi energy and a another maxima at the Fermi energy (HOMO). Both these maxima are also marked by a large DOS from the $\mathrm{Si}$ atoms, indicating a strong mixing between the $\mathrm{Cr} d$ and $\mathrm{Si}$ $s p$ states. As the $\mathrm{CrSi}_{12}$ cluster is deposited on the $\mathrm{Si}(111)$ surface, two situations can arise. In the configuration (I) shown in Fig. 2, the $\mathrm{Cr}$ atom moves down after deposition and is able to bond with $\mathrm{Si}$ atoms at the surface. The resulting DOS is shown in Fig. 4(b) and one again notices that the
$\mathrm{Cr} d$-states hybridize strongly with $\mathrm{Si} s p$ states as both densities show appreciable maxima at similar energies. A different electronic scenario emerges in configuration (III), shown in Fig. 3. The corresponding DOS is shown in Figs. 4(c) and 4(d) for the paramagnetic and magnetic DOS, respectively. In the paramagnetic DOS [shown in Fig. 4(c)], it can be noted that, while the $\mathrm{Cr} d$ states present a maxima at the Fermi energy, the density of $s p$-states from $\mathrm{Si}$ is relatively low. The smaller mixing allows these $\mathrm{Cr} d$ states to retain their atomic unpaired spins and, therefore, the most stable solution for this configuration is a magnetic one, as shown in Fig. 4(d). Hence, the key to magnetism in silicon is to decouple some of the localized transition metal states from the silicon hybridization and the way to accomplish this is to link Si atoms to their own kind. While selected deposition provides such opportunity, one can also accomplish the same effect by thickening the silicon shell, i.e., going to larger silicon species. It is important to note that this is likely the reason why $\mathrm{Cr}$ impurities in bulk silicon have been observed to retain large spin moments. ${ }^{23}$

\section{CONCLUSIONS AND SUMMARY}

The present work shows that, while the individual $\mathrm{CrSi}_{12}$ clusters are quite stable and do not carry any spin moment, the spin moments can emerge when two such clusters are combined to create a composite cluster or when the clusters are randomly deposited on a $\mathrm{Si}(111)$ surface. In real situations, when the clusters are deposited one would expect a combination of the two effects, since a second layer of clusters may deposit on the previous ones or the clusters may coalesce. Since many of these combinations are magnetic, the random cluster deposition appears as an excellent opportunity to synthesize magnetic silicon-based materials. There are already experiments where different clusters are deposited on silicon surfaces. ${ }^{24-26}$ Zilani et al. ${ }^{24}$ have deposited transition metals like Fe. Uchida et al. deposited $\mathrm{TaSi}_{n}$ (Ref. 25) and $\mathrm{MoSi}_{n}$ (Ref. 26) clusters on $\operatorname{Si}(111)-(7 \times 7)$ but, to our knowledge, no experiment has been done pursuing the magnetic properties of these systems. We hope that the present work will stimulate such experiments. On our part, we are currently working with experimental groups to realize such a possibility.

\section{ACKNOWLEDGMENT}

We gratefully acknowledge support from the U.S. Department of the Army through a MURI Grant No. W911NF-06$1-0280$.

\footnotetext{
*snkhanna@vcu.edu

${ }^{1}$ A. W. Castleman, Jr., and S. N. Khanna, J. Phys. Chem. C 113, 2664 (2009).

${ }^{2}$ S. A. Claridge, A. W. Castleman, S. N. Khanna, C. B. Murray, A. Sen, and P. S. Weiss, ACS Nano 3, 244 (2009).

${ }^{3}$ C. Peineke, M. Attoui, R. Robles, A. Reber, S. Khanna, and A.
}

Schmidt-Ott, J. Aerosol Sci. 40, 423 (2009).

${ }^{4}$ J. H. Weaver and D. M. Poirier, Solid State Physics (Academic Press, Boston, 1994), Vol. 48, p. 1.

${ }^{5}$ C. A. Mirkin and W. B. Caldwell, Tetrahedron 52, 5113 (1996).

${ }^{6}$ L. Forro and L. Mihaly, Rep. Prog. Phys. 64, 649 (2001).

${ }^{7}$ M. Qian, S. V. Ong, S. N. Khanna, and M. B. Knickelbein, Phys. 
Rev. B 75, 104424 (2007).

${ }^{8}$ H. Hiura, T. Miyazaki, and T. Kanayama, Phys. Rev. Lett. 86, 1733 (2001).

${ }^{9}$ S. N. Khanna, B. K. Rao, and P. Jena, Phys. Rev. Lett. 89, 016803 (2002).

${ }^{10}$ J. Ulises Reveles and S. N. Khanna, Phys. Rev. B 72, 165413 (2005).

${ }^{11}$ J. Ulises Reveles and S. N. Khanna, Phys. Rev. B 74, 035435 (2006).

${ }^{12}$ C. Xiao, F. Hagelberg, and W. A. Lester, Phys. Rev. B 66, 075425 (2002).

${ }^{13}$ P. Sen and L. Mitas, Phys. Rev. B 68, 155404 (2003).

${ }^{14}$ V. Kumar and Y. Kawazoe, Phys. Rev. Lett. 87, 045503 (2001).

${ }^{15}$ J. Lu and S. Nagase, Phys. Rev. Lett. 90, 115506 (2003).

${ }^{16}$ W. Zheng, J. M. Nilles, D. Radisic, and K. H. Bowen, J. Chem. Phys. 122, 071101 (2005).

${ }^{17}$ L. Kong and J. R. Chelikowsky, Phys. Rev. B 77, 073401
(2008).

${ }^{18}$ P. R. Bandaru, J. Park, J. S. Lee, Y. J. Tang, L. Chen, S. Jin, S. A. Song, and J. R. O’Brien, Appl. Phys. Lett. 89, 112502 (2006).

${ }^{19}$ G. Kresse and J. Furthmller, Comput. Mater. Sci. 6, 15 (1996).

${ }^{20}$ P. E. Blöchl, Phys. Rev. B 50, 17953 (1994).

${ }^{21}$ G. Kresse and D. Joubert, Phys. Rev. B 59, 1758 (1999).

${ }^{22}$ J. P. Perdew, J. A. Chevary, S. H. Vosko, K. A. Jackson, M. R. Pederson, D. J. Singh, and C. Fiolhais, Phys. Rev. B 46, 6671 (1992).

${ }^{23}$ Z. Z. Zhang, B. Partoens, K. Chang, and F. M. Peeters, Phys. Rev. B 77, 155201 (2008).

${ }^{24}$ M. Zilani, Y. Sun, H. Xu, G. Peng, Y. Feng, X. Wang, and A. Wee, Surf. Sci. 601, 2486 (2007).

${ }^{25}$ N. Uchida, L. Bolotov, T. Miyazaki, and T. Kanayama, J. Phys. D 36, L43 (2003).

${ }^{26}$ N. Uchida, H. Yahata, T. Kanayama, and L. Bolotov, Appl. Phys. Lett. 91, 063109 (2007). 